\title{
A Comparative Study of Fractional Order Models on State of Charge Estimation for Lithium lon Batteries
}

\author{
Jinpeng Tian ${ }^{1,2}$, Rui Xiong ${ }^{1 *} \mathbb{D}$, Weixiang Shen ${ }^{2}$ and Ju Wang ${ }^{1}$
}

\begin{abstract}
State of charge (SOC) estimation for lithium ion batteries plays a critical role in battery management systems for electric vehicles. Battery fractional order models (FOMs) which come from frequency-domain modelling have provided a distinct insight into SOC estimation. In this article, we compare five state-of-the-art FOMs in terms of SOC estimation. To this end, firstly, characterisation tests on lithium ion batteries are conducted, and the experimental results are used to identify FOM parameters. Parameter identification results show that increasing the complexity of FOMs cannot always improve accuracy. The model R(RQ)W shows superior identification accuracy than the other four FOMs. Secondly, the SOC estimation based on a fractional order unscented Kalman filter is conducted to compare model accuracy and computational burden under different profiles, memory lengths, ambient temperatures, cells and voltage/current drifts. The evaluation results reveal that the SOC estimation accuracy does not necessarily positively correlate to the complexity of FOMs. Although more complex models can have better robustness against temperature variation, $R(R Q)$, the simplest FOM, can overall provide satisfactory accuracy. Validation results on different cells demonstrate the generalisation ability of FOMs, and $\mathrm{R}(\mathrm{RQ})$ outperforms other models. Moreover, $\mathrm{R}(\mathrm{RQ})$ shows better robustness against truncation error and can maintain high accuracy even under the occurrence of current or voltage sensor drift.
\end{abstract}

Keywords: Electric vehicle, Lithium ion battery, Fractional order model, State of charge

\section{Introduction}

\subsection{Literature Review}

Lithium ion batteries are the paramount component that enables the market penetration of electric vehicles (EVs). As a time-variant electrochemical power source, management of lithium ion batteries has drawn much attention. In particular, the accurate estimation of state of charge (SOC), which directly decides the driving distance and power performance, is one of the most indispensable tasks of battery management systems (BMSs) $[1,2]$.

SOC is generally defined as the ratio between the available capacity and maximum capacity [3]. If a battery is

\footnotetext{
${ }^{*}$ Correspondence: rxiong@bit.edu.cn

1 Department of Vehicle Engineering, School of Mechanical Engineering,

Beijing Institute of Technology, Beijing 100081, China

Full list of author information is available at the end of the article
}

fully charged, its SOC is defined as $100 \%$. If a battery is depleted completely, its SOC is set to $0 \%$. SOC works like a fuel gauge in conventional vehicles but it is more intricate as it cannot be measured directly. Numerous methods for SOC estimation have been proposed and are summarised in recent reviews [1, 4]. Among them, model-based methods are most prevalent since battery models can be combined with observers to provide a closed-loop SOC estimation result [4]. Frequently used models for lithium ion batteries can be categorised into three main groups, namely electrochemical models (EMs), equivalent circuit models (ECMs) and fractional order models (FOMs).

EMs include a set of partial differential equations (PDEs) to describe electrochemical reactions $[5,6]$. Thanks to their explicit physical basis, EMs have been widely employed for the simulation of time consuming 
or dangerous experiments, such as short circuits $[7,8]$ and battery ageing [9-11]. However, there are plenty of parameters in EMs, some of which cannot be obtained without assistance from battery manufacturers [12]. Furthermore, solving PDEs is an expensive task in terms of calculations [13]. These drawbacks hinder onboard applications of EM-based SOC estimation.

ECMs are a popular choice for SOC estimation for lithium ion batteries. They consist of primarily ideal electrical components like capacitance, resistance and voltage source. Therefore they are computationally preferable $[14,15]$ and are widely applied in microcontrollers [1618]. A few comparative studies have been conducted on ECMs to reveal their abilities $[19,20]$. The main demerits of ECMs include low accuracy and weak physical basis.

In the frequency domain, impedance models are widely used to the interpretation of processes of different characteristic frequencies in electrochemical impedance spectra [21]. Studies have demonstrated the effectiveness of the impedance models in the fields of ageing diagnosis $[22,23]$ and the design of heating strategies $[24,25]$. Recently, the impedance models designed to fit impedance spectra in the frequency domain have been transplanted to the time domain for voltage simulation and state estimation. This results in the so-called FOMs featuring constant phase elements (CPEs) which should be interpreted by fractional calculus [26-28]. In 2011, Andre et al. [29] studied the impedance spectra of a lithium ion battery and proposed a FOM containing three CPEs. This FOM was parameterised using EIS before being adopted for voltage simulation. The results showed that it is superior to a third order ECM. Hu et al. [30, 31] integrated this FOM with adaptive fractional order EKFs to estimate SOC while updating part of the model parameters. The results showed the FOM has smaller SOC estimation error than a second order ECM. A different FOM with two CPEs was proposed by $\mathrm{Xu}$ et al. [32] and it was combined with a fractional order EKF to estimate SOC. The comparison with a first order ECM proved that the FOM has better voltage and SOC estimation accuracy. Several observers have also been designed for this FOM to achieve accurate and robust SOC estimation results $[33,34]$. Another FOM with two CPEs was proposed in [35], where the structural relationship between FOMs and ECMs was explained in detail. It was concluded that the first order ECM outperforms the ECM in terms of voltage simulation, even in the presence of short circuit [36]. In Ref. [37], Xu et al. simplified the FOM in Ref. [29] into a FOM with two "Zarc" elements, which corresponds to a parallel connection of a resistance and a CPE. The voltage simulation accuracy of the simplified FOM was illustrated by comparing with that of a second order ECM. This FOM was also adopted in Ref. [38], where a framework for $\mathrm{SOC}$ and state of health $(\mathrm{SOH})$ co-estimation was proposed. Two fractional order EKFs were combined to estimate SOC and battery capacity, respectively. The results showed that the FOM was better than the second order and third order ECMs in terms of SOC and capacity estimation. To facilitate SOC estimation, the FOM was combined with a fractional order UKF in Ref. [39], and a corresponding observer was designed in Ref. [40]. Both studies illustrated improved accuracy compared with conventional ECMs. In Ref. [41], a simplified FOM containing only one CPE was employed for online identification of the open circuit voltage (OCV). Its accuracy was proved to be higher than that of the first order ECM. This conclusion is in agreement with Ref. [42] in which offline comparison of the FOM with five ECMs was conducted. Besides high accuracy, Sutter et al. [43] found the parameters of this FOM are associated with battery ageing and can reflect electrochemical information to a certain extent. Jiang et al. [44] developed a continuous time-domain identification method for this FOM. This model has also been employed for SOC estimation by using different fractional Kalman filters [45, 46]. The results showed that this FOM provides higher accuracy than the first order ECM.

\subsection{Contributions}

Based upon the above discussions, one can note that a number of FOMs have been demonstrated to be more accurate and robust than conventional ECMs not only for voltage simulation but also for SOC estimation. However, different components and structures of FOMs inevitably affect the performance of their applications in voltage simulation and SOC estimation. As pointed out in Ref. [35], the most complex FOM does not necessarily provide the smallest voltage simulation error. Their ability to estimate SOC remains unclear.

In this article, for the first time, we systematically compare five widely used FOMs for SOC estimation of lithium ion batteries. Besides estimation accuracy, the influence of memory length, ambient temperature, cell difference, voltage and current sensor drift is discussed to examine the robustness. This study can provide guidance to researchers and engineers in selecting the best FOM for SOC estimation.

\subsection{Paper Organisation}

The remainder of the present paper is organised as follows. The experimental setup and battery tests are introduced in Section 2. Five FOMs and their identification are outlined in Section 3. The SOC estimation method and comparison of FOMs on SOC estimation are described in Section 4. Finally, conclusions are given in Section 5. 


\section{Experimental Setup and Battery Tests}

In this section, the battery experiments are introduced to construct the dataset for the comparison of FOMs. In this work, seven lithium-manganese-cobalt-oxide (NMC)/graphite batteries randomly selected from the same batch are tested, and their specifications are given in Table 1. Cell 1 is used as the primary cell for comparison and cells 2-7 are used to evaluate the generalisation of FOMs. Figure 1 shows the primary components of the test platform. This platform consists of an ARBIN tester, which is capable of charging/discharging the batteries under the control of the host computer, and a thermal chamber to regulate ambient temperature. The capacity test is first performed to measure battery capacity according to the standard charging/discharging method specified by the battery manufacturer. Then, the hybrid pulse power characterisation (HPPC) test is carried out for parameter identification. It comprises 49 groups of current pulses at the SOCs of $100 \%, 98 \%, \ldots, 4 \%$ in order to consider the SOC dependence of model parameters. The current pulses and corresponding voltage responses at different SOCs are be used for parameter identification of FOMs in Section 3.2. It is followed by two EV driving schedules, namely the dynamic stress test (DST) and urban dynamometer driving schedule (UDDS). The SOC estimation is conducted based on both profiles to

Table 1 Specifications of the NMC lithium ion batteries

\begin{tabular}{lllll}
\hline Specifications & $\begin{array}{l}\text { Nominal } \\
\text { capacity } \\
\text { (A.h) }\end{array}$ & $\begin{array}{l}\text { Nominal } \\
\text { voltage } \\
\text { (V) }\end{array}$ & $\begin{array}{l}\text { Cut-off } \\
\text { voltage } \\
\text { (V) }\end{array}$ & $\begin{array}{l}\text { Maximum } \\
\text { charging/ } \\
\text { discharging } \\
\text { current (A) }\end{array}$ \\
\hline Quantity & 25 & 3.65 & $4.15 / 2.5$ & $75 / 100$ \\
\hline
\end{tabular}

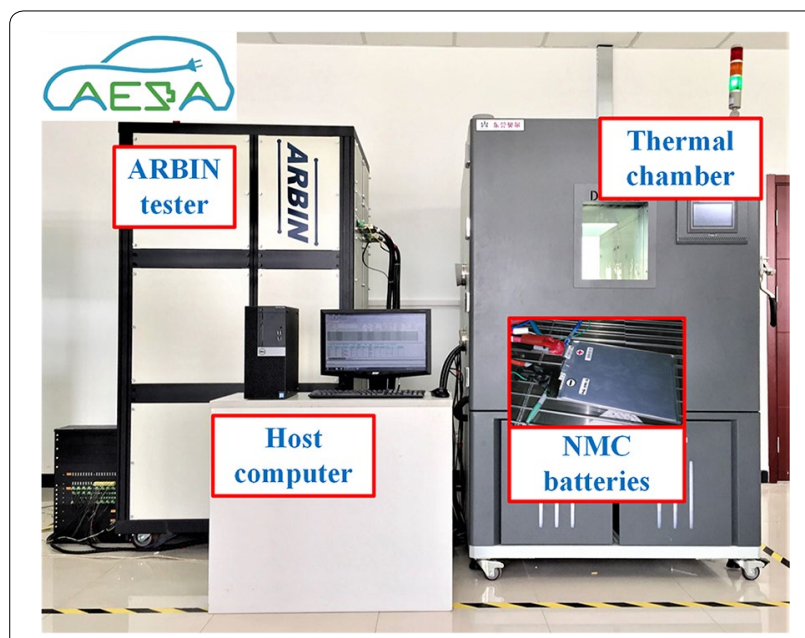

Figure 1 Battery test platform simulate operating conditions in EVs. To assess the performance of FOMs under different conditions, four characterisation tests are conducted at $15^{\circ} \mathrm{C}, 25^{\circ} \mathrm{C}$ and $35^{\circ} \mathrm{C}$. In all tests, the sampling frequency is set to be $1 \mathrm{~Hz}$. The current and voltage profiles of all tests are depicted in Figure 2.

\section{Fractional Order Models}

\subsection{Model Structures}

A few FOMs that were developed for fitting the impedance spectra have been introduced to time-domain applications in BMSs. Figure 3 provides a summary of the widely used five FOMs in the literature for voltage simulation and SOC estimation. In these models, $U_{\mathrm{oc}}$ stands for OCV. $R_{\mathrm{i}}, R_{1}$ and $R_{2}$ represent resistances. $U_{\mathrm{t}}$ and $I$ denote terminal voltage and current, respectively. $I$ is defined as positive for battery charging. The constant phase element (CPE) is a fractional order element whose impedance at frequency $f$ is expressed as

$$
Z_{\mathrm{CPE}}(\omega)=\frac{1}{Q(\mathrm{j} 2 \pi f)^{\alpha}}, \quad 0<\alpha<1,
$$

where $Q\left(s^{\alpha} / \Omega\right)$ and $\alpha$ are its coefficient and order, respectively. The CPE is a generalised element which can represent the ideal capacitance $(\alpha=1)$ and resistance $(\alpha=0)$. It is of interest to note that the serially connected CPE is often referred to as the Warburg element, a specific CPE with a fixed order of 0.5. In this article, this name is adopted to make it distinguishable but its order is considered as a variable therefore it is as flexible as the CPE. Its coefficient and order are defined as $W$ and $\beta$, respectively. For clarity, the models are named according to the model

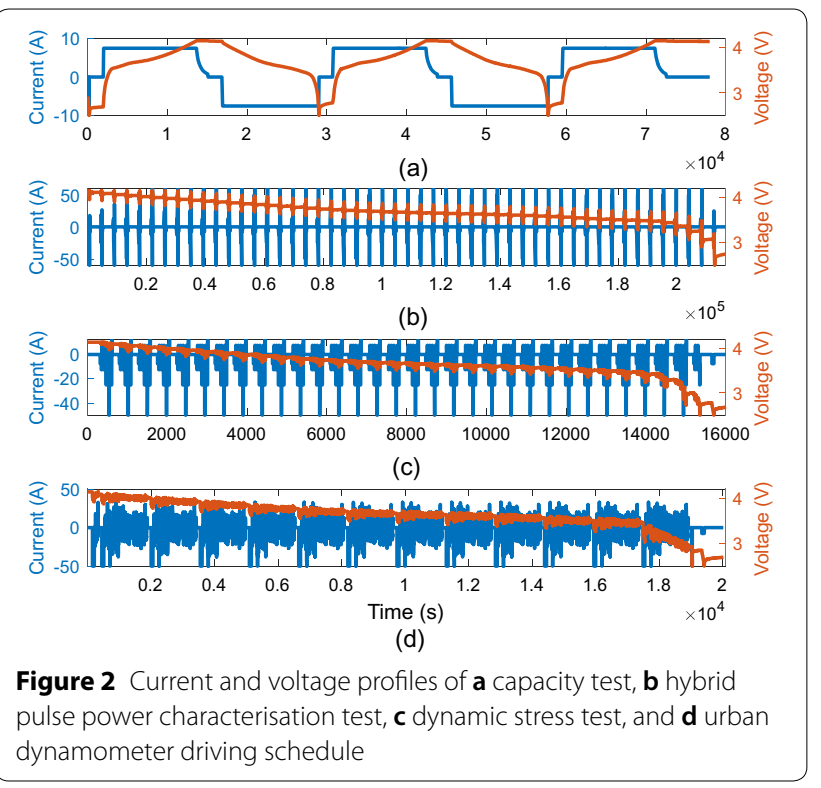




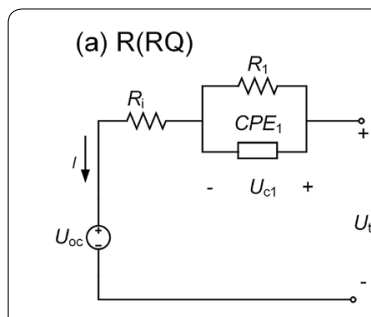

(c) $R(R W Q)$

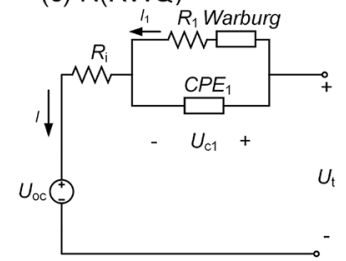

(e) $R(R Q)(R Q) W$ (b) $R(R Q) W$

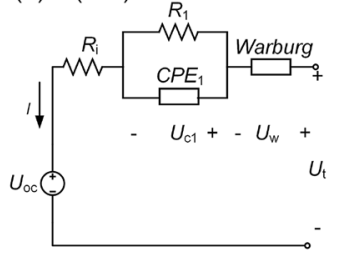

(d) $\mathrm{R}(\mathrm{RQ})(\mathrm{RQ})$
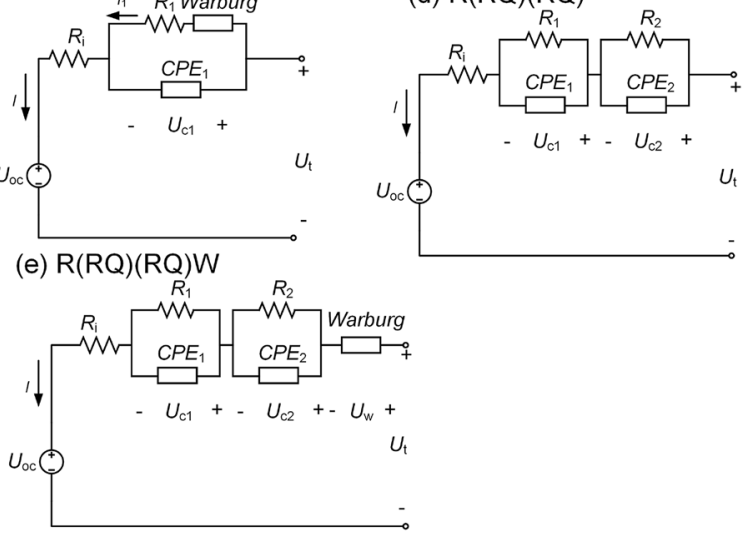

Figure 3 Five fractional order models reported in the literature

structure. For example, R(RQ)W represents a model which consists of a resistance $R$, a R//CPE parallel branch and a Warburg element connected in series.

The voltage $U_{\mathrm{c}}$ across a $\mathrm{R} / / \mathrm{CPE}$ branch and $U_{\mathrm{w}}$ across a Warburg element are governed by

$$
\left\{\begin{array}{l}
\tau \mathrm{D}^{\alpha} U_{\mathrm{c}}(t)=R I(t)-U_{\mathrm{c}}(t) \\
W \mathrm{D}^{\beta} U_{\mathrm{W}}(t)=I(t)
\end{array}\right.
$$

where $\tau=R Q$ stands for a model parameter similar to a time constant. $\mathrm{D}^{\alpha} f(t)$ represents the Grünwald-Letnikov derivative [47] of a function $f(t)$, which provides an intuitive discretised form:

$$
\left\{\begin{array}{r}
\mathrm{D}^{\alpha} f(t)=\lim _{h \rightarrow 0} \frac{1}{h^{\alpha}} \sum_{i=0}^{\left[\frac{t}{h}\right]}(-1)^{i}\left(\begin{array}{c}
\alpha \\
i
\end{array}\right) f(t-i h), \\
\left(\begin{array}{c}
\alpha \\
i
\end{array}\right)=\left\{\begin{array}{cc}
\frac{(\alpha-(i-1)) \cdots(\alpha-1) \alpha}{i !}, & i>0 \\
1, & i=0,
\end{array}\right.
\end{array}\right.
$$

where $h$ denotes the step length and is set to $1 \mathrm{~s}$ in this work. $[t / h]$ gives the greatest integer less than or equal to $t / h$. It is evident that in the time domain, the fractional order $\alpha$ leads to a memory of all values of $f(t)$ in the range of $[0, t]$. In order to reduce the computational cost, a short memory principle [47] is applied, and only data in the range of $[t-L, t]$ are taken into account:

$$
\mathrm{D}^{\alpha} f(t) \approx \frac{1}{h^{\alpha}} \sum_{i=0}^{L}(-1)^{i}\left(\begin{array}{c}
\alpha \\
i
\end{array}\right) f(t-i h) .
$$

where $L$ denotes the memory length, and it is set to 20 in this work. The five models in Figure 3 have been applied for SOC estimation based on sampled current and voltage data. To this end, these models should be firstly discretised. In light of the Kirchhoff's circuit laws and Eqs. (2)-(4), the discretised equations of the five FOMs are presented in Table 2, in which a new symbol is defined as

$$
\mathrm{D}^{(\alpha)} f(t)=\frac{1}{h^{\alpha}} \sum_{i=1}^{L}(-1)^{i}\left(\begin{array}{c}
\alpha \\
i
\end{array}\right) f(t-i h)
$$

for brevity. The state space for SOC estimation is elaborated in Section 4.

\subsection{Parameter Identification}

In this subsection, the model parameter identification is performed for five FOMs based on the HPPC data obtained at $25^{\circ} \mathrm{C}$, where the current pulses are imposed at each SOC and the sampled current serves as the input of the FOM to simulate voltage response. The parameters of the FOMs are obtained when the root mean square error (RMSE) is minimised between the measured voltage $U_{\mathrm{t}}$ and FOM output $\widehat{U}_{\mathrm{t}}$. The RMSE is expressed as

$$
R M S E=\sqrt{\frac{1}{N} \sum_{i=1}^{N}\left(U_{\mathrm{t}}(k)-\hat{U}_{\mathrm{t}}(k)\right)^{2}},
$$

where $N$ is the total number of $U_{\mathrm{t}}$. The maximum absolute error (MAE) is taken as another index to evaluate the identification accuracy, but it is not considered as an objective of identification. It is expressed as

$$
M A E=\max \left(\left|U_{\mathrm{t}}(k)-\hat{U}_{\mathrm{t}}(k)\right|\right), \quad 1 \leq k \leq N .
$$

The FOM parameters that need to be identified are listed in Table 2. It should be emphasised that although $U_{\text {oc }}$ can be measured through OCV tests [48], it is considered as a model parameter to be identified in this study since it has proved that OCV identification can help to improve SOC and SOH estimation accuracy $[41,49]$.

\subsubsection{Parameter Identification Method}

Due to the intrinsic nonlinearity caused by fractional calculus, the RMSE is usually minimised by evolutionary algorithms such as the genetic algorithm (GA). Such algorithms solve optimisation problems by searching for solutions in a given parameter range. Hence, they are 


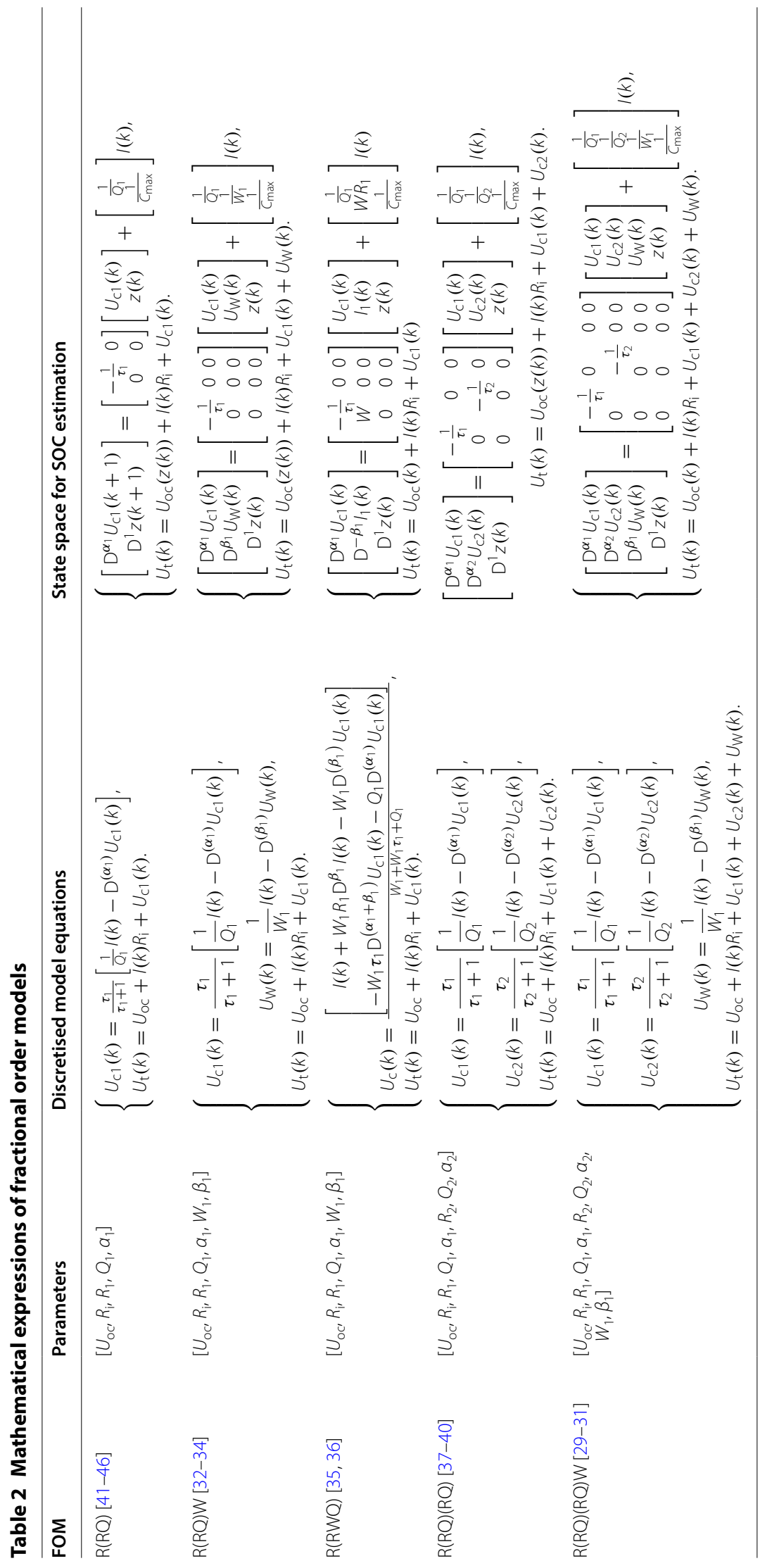


Table 3 The main procedures of the parameter identification method

Step 1: Present the range of nonlinear parameters and stopping criteria.

Step 2: Randomly generate the population in the range.

Step 3: Generate the $U_{c}$ and/or $U_{w}$ vectors using discretised model equations in Table 2.

Step 4: Identify $\left[U_{\text {oc }} R_{\mathrm{i}}\right]$ using least squares and output the RMSE.

Step 5: Evaluate the stopping criteria. If the criteria are met, stop and return the best solution. If not, go to the next step.

Step 6: Generate a new population by crossover, mutation and selection. Go to Step 3

not efficient for linear optimisation. On the other hand, $\left[U_{\mathrm{oc}}, R_{\mathrm{i}}\right]$ can be easily identified by the least squares (LS) algorithm when the polarisation voltage $U_{\mathrm{c}}$ and $U_{\mathrm{w}}$ are determined using the recursive equations in Table 2. Accordingly, GA is used to optimise the other nonlinear parameters while the LS is embedded in the cost function to identify linear parameters $\left[U_{\mathrm{oc}}, R_{\mathrm{i}}\right]$ and compute the RMSE. Its main procedures are listed in Table 3. This identification method has been proven to be more efficient than a conventional GA in Ref. [49] as it reduces the number of parameters to be identified through GA while guaranteeing the globally optimal solutions.

\subsubsection{Parameter Identification Results}

As mentioned previously, the HPPC test consists of current pulses over the SOC range of [4\%, 100\%] with an interval of $2 \%$. Totally 49 groups of model parameters are identified for each FOM. Take the R(RQ)(RQ)W as an example, the model parameters identified at $\mathrm{SOC}=50 \%$ are listed in Table 4, and the measured and simulated voltage are plotted together with the current pulses in Figure 4. It is evident that the simulated voltage is close to the experimental data with the RMSE and MAE being $1.012 \mathrm{mV}$ and $4.306 \mathrm{mV}$, respectively, indicating high identification accuracy.

Figure 5 compares the identification accuracy of five FOMs. Overall, the identification error can be grouped into three ranges, namely the SOC ranges of $[60 \%, 100 \%],[20 \%, 60 \%]$ and $[4 \%, 20 \%]$, corresponding to the high, middle and low SOC ranges, respectively. In high SOC range, both the RMSE and MAE of five FOMs show visible fluctuations, with the maximum $\mathrm{RMSE}=2.57 \mathrm{mV}$ and the maximum $\mathrm{MAE}=$ $11.73 \mathrm{mV}$. When the SOC decreases to [20\%, 60\%], the fluctuations fade down, and the maximum RMSE and maximum MAE decrease to $1.50 \mathrm{mV}$ and $6.01 \mathrm{mV}$, respectively. In these ranges, all models show a similar
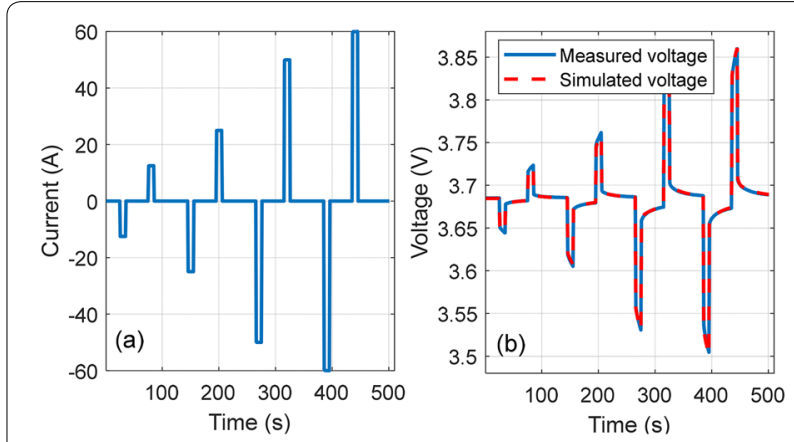

Figure 4 Current and voltage at $\mathrm{SOC}=50 \%$ : a Current pulses, b Measured voltage and simulated voltage based on $\mathrm{R}(\mathrm{RQ})(\mathrm{RQ}) \mathrm{W}$

accuracy level. When the SOC further decreases to [4\%, $20 \%]$, the accuracy of all FOMs decreases significantly. It can be observed from Figure 5(b) that the maximum and minimum RMSE are $8.76 \mathrm{mV}$ and $5.44 \mathrm{mV}$, respectively, which shows a similar trend with the MAE shown in Figure 5(d).

It can also be seen from Figure 5 that the simplest model $R(R Q)$ has the largest error and this error is attenuated by adding a Warburg element, i.e., $\mathrm{R}(\mathrm{RQ})$ $\mathrm{W}$ and R(RWQ). The FOMs with two R//CPE branches including $R(R Q)(R Q)$ and $R(R Q)(R Q) W$ are more complex than the other FOMs but they do not necessarily provide a smaller mean and average RMSE or MAE. On the contrary, they are inferior to $\mathrm{R}(\mathrm{RQ}) \mathrm{W}$ and $\mathrm{R}(\mathrm{RWQ})$ in the low SOC region. This result shows the identification accuracy is not positively related to the complexity of FOMs. Besides, R(RQ)W enjoys smaller average and minimum RMSE and MAE than R(RWQ). In addition, the maximum error of $R(R Q) W$ is comparable to that of $R(R W Q)$ and is much lower than that of remaining models. Thus, $\mathrm{R}(\mathrm{RQ}) \mathrm{W}$ outperforms the other FOMs in terms of identification accuracy.

Table 4 Model parameters of $R(R Q)(R Q) W$ for voltage simulation at $S O C=50 \%$

\begin{tabular}{llllllllll}
\hline$U_{\text {oc }}(\mathrm{V})$ & $R_{\mathrm{i}}(\mathrm{m} \Omega)$ & $R_{\mathbf{1}}(\mathrm{m} \boldsymbol{\Omega})$ & $\boldsymbol{\alpha}_{\mathbf{1}}$ & $Q_{\mathbf{1}}\left(s^{\boldsymbol{\alpha}_{1}} / \boldsymbol{\Omega}\right)$ & $R_{\mathbf{2}}(\mathrm{m} \boldsymbol{\Omega})$ & $\boldsymbol{\alpha}_{\mathbf{2}}$ & $\boldsymbol{Q}_{\mathbf{2}}\left(s^{\left.\boldsymbol{\alpha}_{2} / \boldsymbol{\Omega}\right)}\right.$ & $\boldsymbol{\beta}_{\mathbf{1}}$ & $W_{\mathbf{1}}\left(s^{\boldsymbol{\beta}_{1}} / \boldsymbol{\Omega}\right)$ \\
\hline 3.676 & 2.557 & 19.928 & 0.434 & 18997.936 & 97.051 & 0.863 & 29179.723 & 0.601 & 27330.045 \\
\hline
\end{tabular}



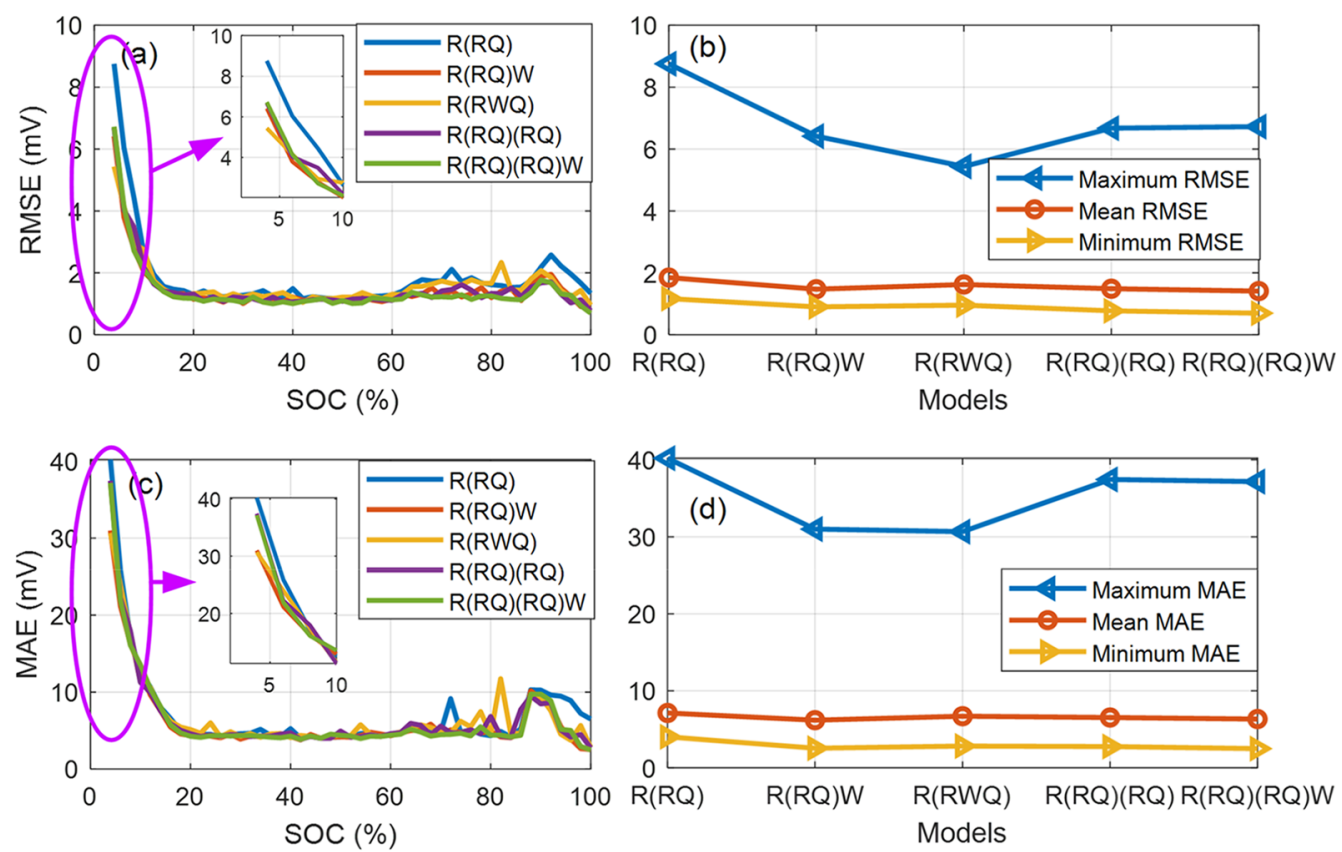

Figure 5 Identification error of 5 FOMs: a RMSE over the SOC range of [4\%, 100\%], b Maximum, mean and minimum RMSE, c The MAE over the SOC range of [4\%, 100\%], d Maximum, mean and minimum MAE

\section{Comparative Analysis of State of Charge Estimation}

\subsection{State of Charge Estimation Method}

SOC estimation based on FOMs has been realised by variants of Kalman filters. To link the output of FOMs and SOC, when a SOC estimation is obtained, the FOM parameters are computed through linear interpolation based on the identified model parameters at 49 SOC points. Furthermore, the relationship between measured current and SOC is established by the Ampere hour counting method, and it is expressed as

$$
\mathrm{D}^{1} z(t)=\frac{\eta}{C_{\max }} I(t)
$$

where $z$ denotes SOC, $\eta$ is the coulombic efficiency which is set to 1 for lithium ion batteries. $C_{\max }$ is the maximum battery capacity determined by the capacity test. By integrating the FOMs and the Ampere hour counting method, state space equations for SOC estimation are established and discretised in Table 2.

Since the FOMs feature fractional order derivatives, conventional Kalman filters are generalised to fractional order ones. In this study, we choose the fractional order unscented Kalman filter (FOUKF) in Ref. [50], which was proved to be able to provide satisfying accuracy as it guarantees a third order approximation of nonlinear functions through unscented transformation. Singular value decomposition is applied to further improve the stability of FOUKF [50]. Its main steps are listed in Table 5.

\subsection{State of Charge Estimation Accuracy}

In this section, the SOC estimation accuracy of five FOMs is compared under the DST and UDDS profiles at $25{ }^{\circ} \mathrm{C}$. To evaluate SOC estimation results, the reference SOC is obtained using the Ampere hour counting method as the initial SOC can be accurately set to $100 \%$ when the battery is fully charged. All filters are set to start $2000 \mathrm{~s}$ after the commencement of the profiles to make sure that their convergence is not influenced by the pre-set SOC limits, i.e., [0,100\%]. Before conducting the SOC estimation, the settings of all filters are calibrated based on the 2000-5000 s of the DST profile. Specifically, the initial value of the state error covariance $P$ is set to be $5 \times 10^{-3} \times \operatorname{diag}(n)$, where $\operatorname{diag}(n)$ stands for an $n \times n$ identify matrix, $n$ is the length of the state vector $x$. The process noise and measurement noise variances $Q$ and $R$ are set to be $10^{-q} \times \operatorname{diag}(n)$ and $10^{-r}$, respectively, with $q, r \in\{0,1,2, \ldots, 20\}$. All combinations of $q$ and $r$ are tried to generate the SOC estimation results of five FOMs, and the combination which provides the minimum RMSE is selected to parameterise $Q$ and $R$ for subsequent SOC estimation. The final settings of each filter are indicated in Table 6.

The SOC estimation results against DST and UDDS profiles are shown in Figure 6. All estimation results 


\section{Table 5 Main procedures of the fractional order unscented Kalman filter}

\section{Step 1 A fractional order system:}

$\left\{D^{\gamma} x_{k+1}=f\left(x_{k}, u_{k}\right)+w_{k}\right.$

$\left\{y_{k}=g\left(x_{k}\right)+v_{k}\right.$,

where $x$ is the state vector, $y$ is the measurement, $u$ is the input. $w$ and $v$ represent the process noise and measurement noise with their variance being $Q$ and $R$, respectively. The state error covariance is defined as $P$.

\section{Step 2 Initialisation:}

Pre-set $Q, R$ and the initial values of $x$ and $P$.

\section{Step 3 Time update:}

(a) Compute sigma points

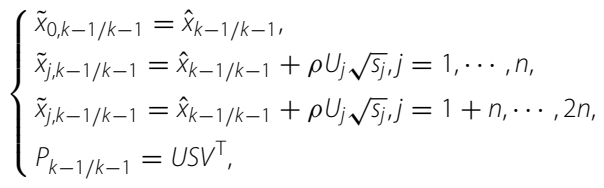

where $n$ is the length of $x . s_{j}$ and $U_{j}$ denote the jth element and jth column of $S$ and $U$, respectively. $\rho$ is a constant in the range of $[1, \sqrt{2}$. (b) Prior state estimation

$$
\left\{\begin{array}{l}
D^{\gamma} \hat{x}_{k / k-1} \approx \sum_{j=0}^{2 n} W_{i}^{m} f\left(x_{j, k-1 / k-1}, u_{k-1}\right), \\
\hat{x}_{k / k-1}=D^{\gamma} \hat{x}_{k / k-1}-\sum_{j=1}^{k}(-1)^{j} \gamma_{j} \hat{x}_{k-1 / k-1}, \\
P_{k / k-1}^{\mathrm{DD}} \approx \sum_{j=0}^{2 n} W_{i}^{c}\left[f\left(\tilde{x}_{j, k-1 / k-1}, u_{k-1}\right)-\mathrm{D}^{\gamma} \hat{x}_{k / k-1}\right] \\
\times\left[f\left(\tilde{x}_{j, k-1 / k-1}, u_{k-1}\right)-\mathrm{D}^{\gamma} \hat{x}_{k / k-1}\right]^{\top}+Q_{1} \\
P_{k / k-1}^{x \mathrm{D}} \approx \sum_{j=0}^{2 n} W_{i}^{c}\left[f\left(\tilde{x}_{j, k-1 / k-1}, u_{k-1}\right)-\mathrm{D}^{\gamma} \hat{x}_{k / k-1}\right] \\
\times\left[f\left(\tilde{x}_{j, k-1 / k-1}, u_{k-1}\right)-\mathrm{D}^{\gamma} \hat{x}_{k / k-1}\right]^{\top}, \\
P_{k / k-1}=P_{k / k-1}^{\mathrm{DD}}+\gamma_{1} P_{k / k-1}^{x \mathrm{D}}+P_{k / k-1}^{\mathrm{Dx}} \gamma_{1}+\sum_{j=1}^{k} \gamma_{j} P_{k-1 / k-1} \gamma_{j} .
\end{array}\right.
$$

where the associated weights are computed as

$$
\left\{\begin{array}{l}
W_{0}^{m}=\frac{\lambda}{\lambda+n^{\prime}} \\
W_{0}^{c}=\frac{\lambda}{\lambda+n}+\left(1-\alpha_{w}^{2}+\beta_{w}\right), \\
W_{i}^{m}=W_{i}^{(c)}=\frac{1}{2(\lambda+n)}, i=1, \ldots, 2 n,
\end{array}\right.
$$

with $\alpha_{\mathrm{w}}$ and $\beta_{\mathrm{w}}$ being two algorithm parameters.

\section{Step 4 Measurement update:}

(a) Create new sigma points using $P_{k / k-1}$

(b) Generate the estimated $y_{k}$ and the corresponding covariance

$$
\left\{\begin{array}{l}
\hat{y}_{k / k-1}=\sum_{j=0}^{2 n} W_{i}^{m} h\left(\tilde{x}_{j, k / k-1}\right), \\
P_{k / k-1}^{y y}=\sum_{j=0}^{2 n} W_{i}^{c}\left[h\left(\tilde{x}_{j, k / k-1}\right)-\hat{y}_{k / k-1}\right]\left[h\left(\tilde{x}_{j, k / k-1}\right)-\hat{y}_{k / k-1}\right]^{\top}+R, \\
P_{k / k-1}^{x y}=\sum_{j=0}^{2 n} W_{i}^{c}\left[h\left(\tilde{x}_{j, k / k-1}\right)-\hat{y}_{k / k-1}\right]\left[h\left(\tilde{x}_{j, k / k-1}\right)-\hat{y}_{k / k-1}\right]^{\top} .
\end{array}\right.
$$

(c) Update the posterior estimation

Step 5: $k=k+1$. Go to Step 3 
Table 6 Settings of five fractional order unscented Kalman filters

\begin{tabular}{llll}
\hline Model & $\boldsymbol{q}$ & $\boldsymbol{r}$ & $\boldsymbol{n}$ \\
\hline $\mathrm{R}(\mathrm{RQ})$ & 9 & 15 & 2 \\
$\mathrm{R}(\mathrm{RQ}) \mathrm{W}$ & 3 & 15 & 3 \\
$\mathrm{R}(\mathrm{RWQ})$ & 8 & 15 & 3 \\
$\mathrm{R}(\mathrm{RQ})(\mathrm{RQ})$ & 8 & 15 & 3 \\
$\mathrm{R}(\mathrm{RQ})(\mathrm{RQ}) \mathrm{W}$ & 8 & 15 & 4 \\
\hline
\end{tabular}

quickly converge to a $\pm 5 \%$ error boundary within $30 \mathrm{~s}$. For the DST profile, $\mathrm{R}(\mathrm{RWQ})$ shows the highest accuracy with the RMSE of only $0.2 \%$, but it fails to achieve high accuracy for the UDDS profile, and its RMSE increases to $1.75 \%$, as shown in Figure 7. Such dispersion also happens to R(RQ)W, whose RMSE is $1.64 \%$ and $2.21 \%$ for the DST and UDDS profiles, respectively. In contrast, the RMSE of the other models is less dependent on profiles.
It implies the high reliability of these models. Moreover, $\mathrm{R}(\mathrm{RQ})$ shows an overall RMSE of $0.57 \%$, which is superior to the other models in terms of SOC estimation accuracy.

\subsection{Robustness of Fractional Order Models 4.3.1 Influence of Memory Length}

One of the most salient features of FOMs is the memory effect, which is described by the Grünwald-Letnikov definition shown in Eq. (3). In real applications, the short memory principle indicated in Eq. (4) is usually applied to prevent the calculation from increasing along with time. To evaluate the influence of memory length on SOC estimation, we adjust the memory length from 5 to 50 at an interval of 5 , and their RMSEs are shown in Figure 8. It is noticeable that for both profiles $R(R Q), R(R Q)(R Q)$ and $\mathrm{R}(\mathrm{RQ})(\mathrm{RQ}) \mathrm{W}$ show consistent behaviours in RMSEs while the other two models show different behaviours. This phenomenon coincides with the profile-dependence observed in Figure 7. Generally speaking, all the

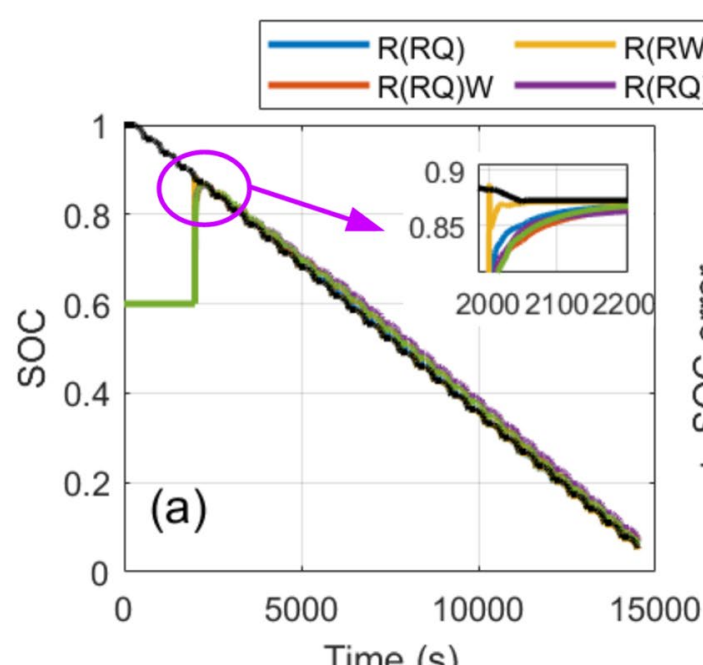

$\begin{array}{ll}\text { RQ) } & R(R Q)(R Q) W \\ \text { Reference }\end{array}$
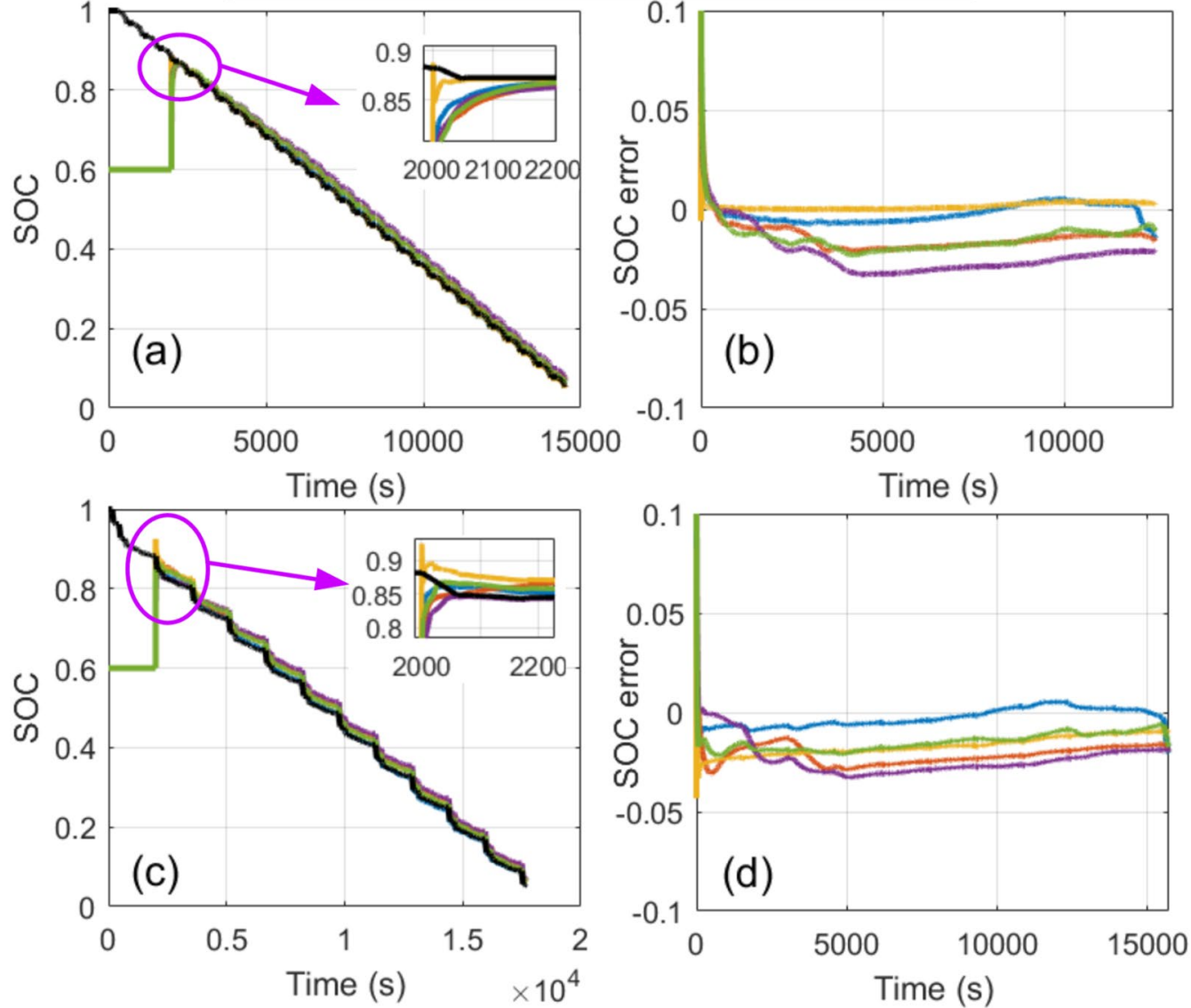

Figure 6 Estimation results of 5 FOMs: a SOC estimation results for DST profile, $\mathbf{b}$ SOC estimation error for DST profile, $\mathbf{c}$ SOC estimation results for UDDS profile, d SOC estimation error for UDDS profile 


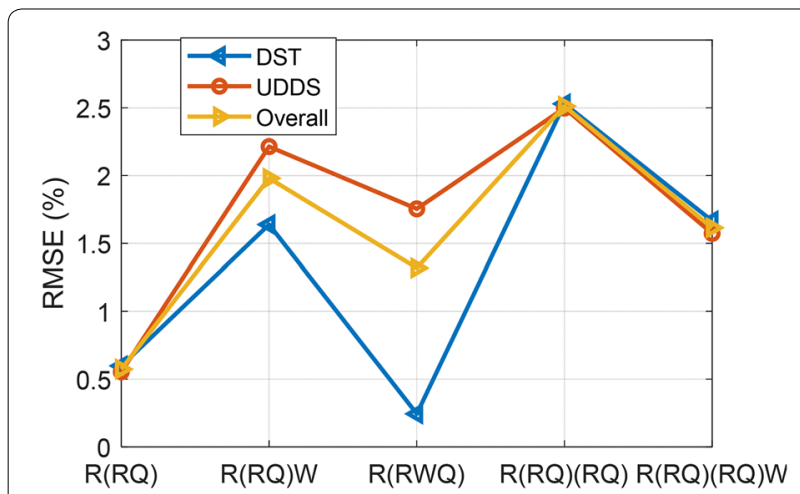

Figure 7 RMSE of five FOMs

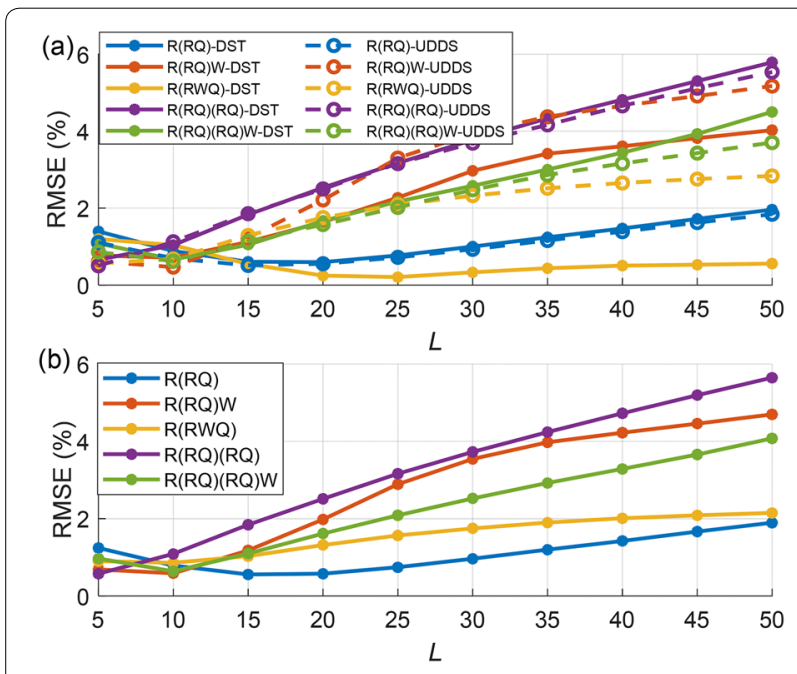

Figure 8 Influence of memory length on SOC estimation errors: a RMSEs for DST and UDDS profiles, $\mathbf{b}$ Overall RMSEs for two profiles

models fail to achieve the minimum RMSE when $L=20$, the memory length defined before parameter identification. For instance, $R(R Q)$ reaches the minimum RMSE of $0.55 \%$ at $L=15$ while $\mathrm{R}(\mathrm{RQ})(\mathrm{RQ}) \mathrm{W}$ reaches the minimum RMSE of 0.64 at $L=10$. To give a more intuitive view, Figure 9 shows the variation of SOC estimation errors of the three models when $L$ increases. A general trend is that the SOC estimation error moves downward as $L$ increases. Therefore, the minimum RMSE is achieved when the SOC error is close to $\mathrm{x}$-axis. This behaviour can be attributed to the truncation error caused by the short memory principle. It can be written as

$$
e(L)=\frac{1}{h^{\alpha}} \sum_{L+1}^{\left[\frac{t}{h}\right]}(-1)^{i}\left(\begin{array}{c}
\alpha \\
i
\end{array}\right) f(t-i h) .
$$
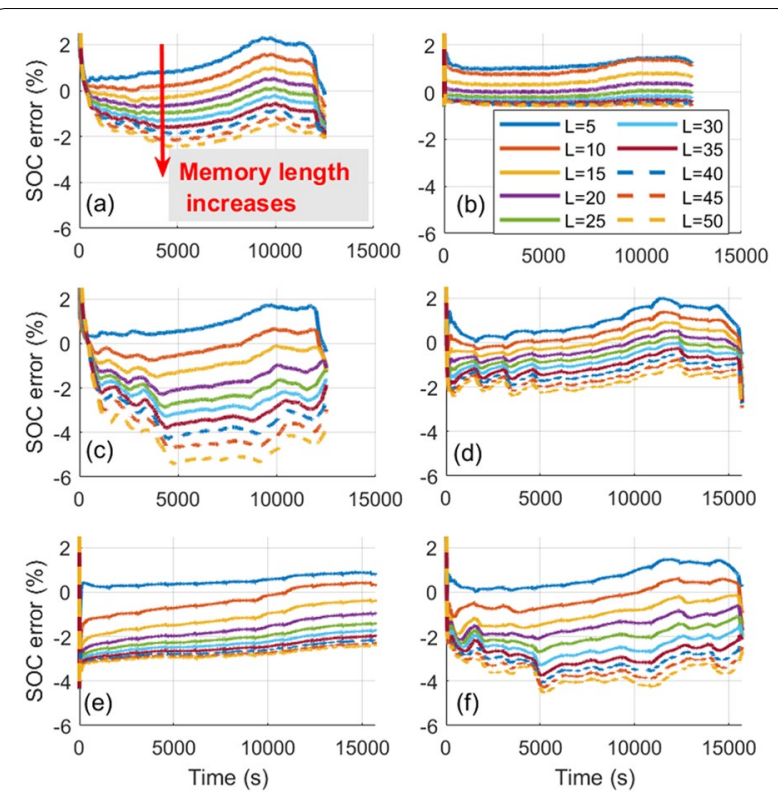

Figure 9 SOC estimation errors under different memory lengths: a $R(R Q)$ for DST profile, $\mathbf{b} R(R W Q$ ) for DST profile, $\mathbf{c}(R Q)(R Q)$ W for DST profile, $\mathbf{d} R(R Q)$ for UDDS profile, e $R(R W Q)$ for UDDS profile, $\mathbf{f} R(R Q)$ (RQ)W for UDDS profile

In the FOMs, polarisation voltages $U_{\mathrm{c}}$ and $U_{\mathrm{w}}$ are depicted by fractional derivatives, therefore the truncation error is dependent on the models and profiles, which violates the assumption of Kalman filters that the errors are white noise [51]. The variances set for a fixed $L$ can be inaccurate when $L$ varies, this will affect the ability of the FOMs to adjust SOC estimation results. Moreover, the FOM with the complex structure is more vulnerable. The RMSE of $\mathrm{R}(\mathrm{RQ})$ changes within $1.34 \%$ while the maximum RMSE of $R(R Q)(R Q)$ changes within $5.08 \%$. In addition, one can see from Figure 10 that the overall computational time almost linearly increases along with the increase of the memory length. Therefore, adjusting the memory length during actual applications can balance computational burdens and estimation accuracy when necessary. For instance, when other computationally intensive tasks like $\mathrm{SOH}$ estimation is in process, the memory length can be reduced.

\subsubsection{Influence of Ambient Temperature}

Battery impedance and OCV are significantly affected by ambient temperature [52]. Thus, it important to evaluate the influence of ambient temperature on SOC estimation accuracy. The filters designed under $25^{\circ} \mathrm{C}$ are applied to estimate the SOC under DST and UDDS profiles at the temperatures of $15{ }^{\circ} \mathrm{C}$ and $35{ }^{\circ} \mathrm{C}$, respectively, and the overall RMSEs are reported in Figure 11. The RMSE of 


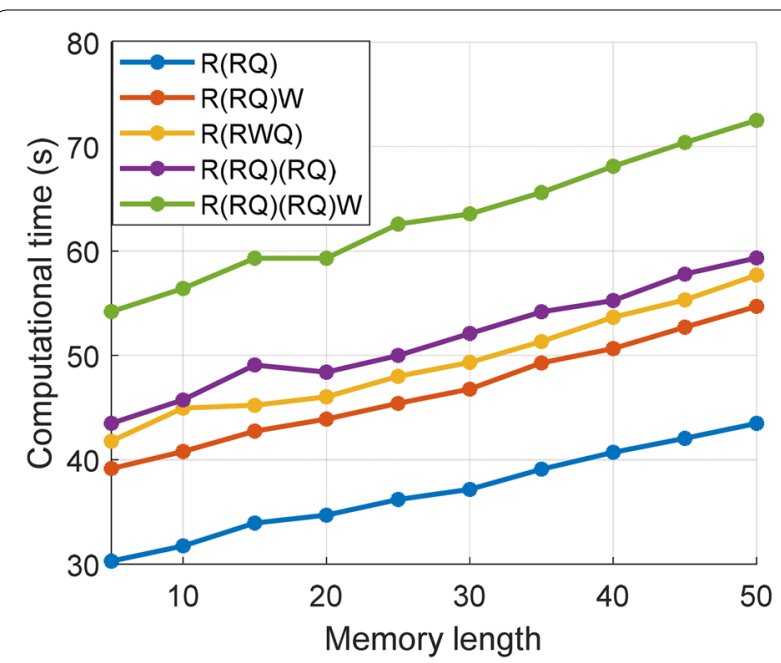

Figure 10 Overall computational times of $\mathrm{SOC}$ estimation under different memory lengths

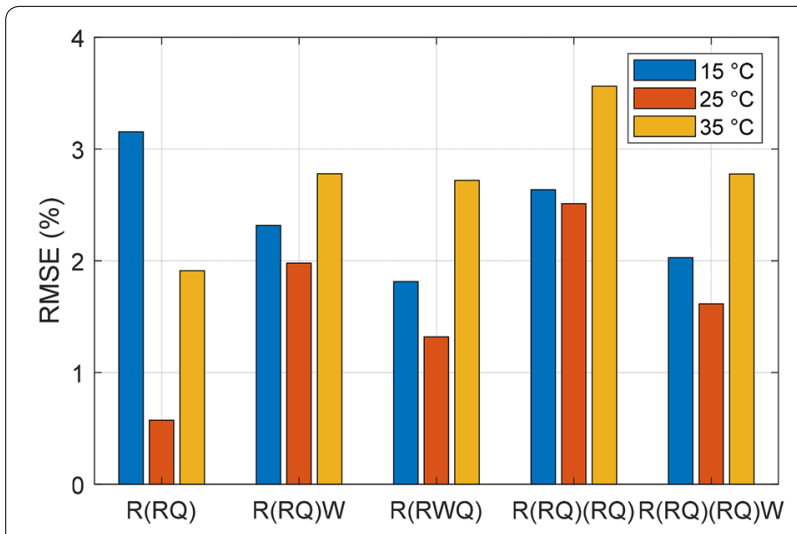

Figure 11 RMSE under different temperatures

all models increases when temperature varies. All models can guarantee a SOC estimation error less than $4 \%$ in case of the temperature variation of $\pm 10{ }^{\circ} \mathrm{C}$. Furthermore, $\mathrm{R}(\mathrm{RQ}) \mathrm{W}, \mathrm{R}(\mathrm{RWQ})$ and $\mathrm{R}(\mathrm{RQ})(\mathrm{RQ}) \mathrm{W}$ can provide a SOC estimation result with the error less than $3 \%$. It is evident that $\mathrm{R}(\mathrm{RQ})$ is most susceptible to temperature variation, and its RMSE increases by 4.94 times when the temperature decreases to $15{ }^{\circ} \mathrm{C}$. In contrast, the RMSEs of the other four FOMs only increase by no more than $106 \%$ as a result of the temperature variation of $\pm 10{ }^{\circ} \mathrm{C}$. Hence, complex FOMs are more robust than the simplest $\mathrm{R}(\mathrm{RQ})$ against temperature variation.

\subsubsection{Influence of Cell Difference}

A battery system for EVs comprises thousands of cells. Modelling cells individually can be computationally demanding [53]. Considering the improved battery manufacturing and screening processes, we can expect to apply a model developed based on one cell to monitor the state of other cells. In this regard, a battery model with high generalisation is desirable to alleviate calculational burdens with ensured performance. To test the generalisation of five FOMs for different cells, the parameterised FOMs are applied to estimate SOC for cells 2 to 7 . The RMSEs and the standard deviations (SDs) of the RMSEs are shown in Figure 12. It can be noted all models show satisfying generalisation ability when being applied for different cells with the RMSE less than 2.38\%. The R(RQ) has the highest estimation accuracy and smallest SD while the most complex models $R(R Q)(R Q)$ and $R(R Q)$ (RQ)W show the highest deviation, as can be observed from the RMSEs of cells 6 and 7.

\subsubsection{Influence of Voltage Sensor Drift}

In real applications where battery voltage is sampled by a BMS, the measurement accuracy of the BMS cannot be as high as that of the test platform. Furthermore, as discussed by Zheng et al. [4, 20], voltage drift is more detrimental to SOC estimation than random noise, although its magnitude is generally within $5 \mathrm{mV}$ [54]. In this subsection, the voltage drift is set to $[-10,10] \mathrm{mV}$ with an interval of $2 \mathrm{mV}$ in order to evaluate the robustness of five FOMs, and their RMSEs for the two profiles are shown in Figure 13. It can be observed that the influence of voltage drift on RMSE qualitatively agrees with that of memory length reported in Figure 8. The RMSEs of R(RQ)(RQ), $R(R Q) W$ and $R(R Q)(R Q) W$ show an approximately linear relationship with voltage drift while $R(R Q)$ and $R(R W Q)$ have a minimum RMSE in the wide range of voltage drift. Over the voltage drift range of $[-10,10] \mathrm{mV}$, the maximum RMSE of all five FOMs is within 3.40\%, indicating a small influence of voltage drift on SOC estimation accuracy. Furthermore, all the FOMs have a similar gradient for positive voltage drift. Since R(RQ) has the minimum original RMSE but has the largest increase in relative

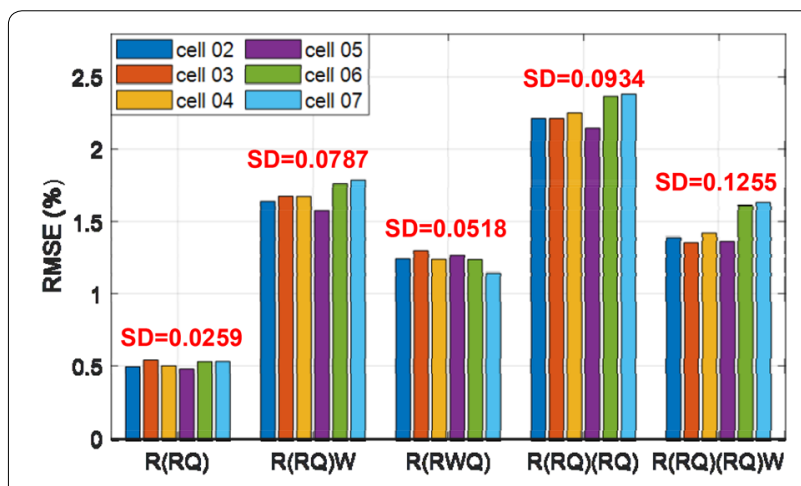

Figure 12 RMSE and standard deviation for the estimation results of different cells 


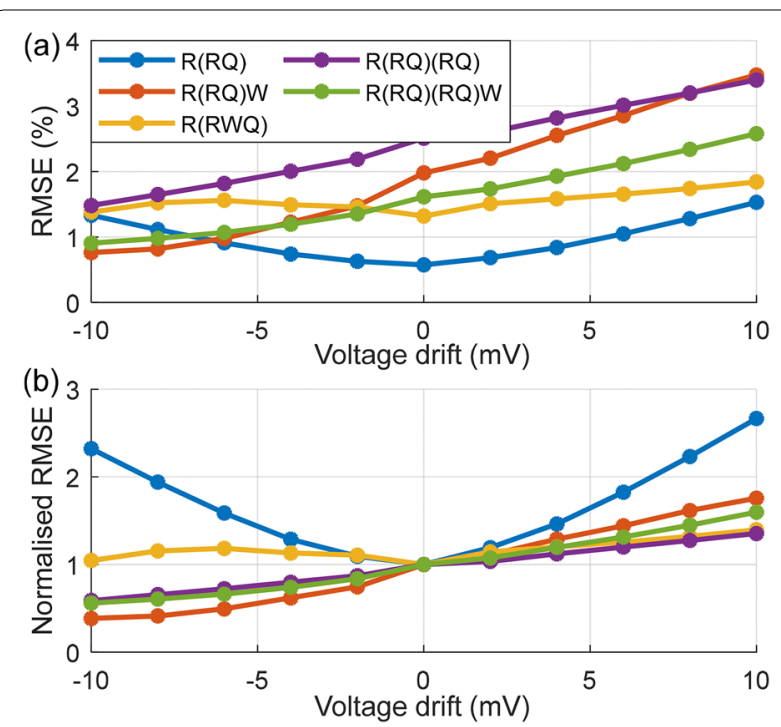

Figure 13 Influence of voltage drift on SOC estimation error: a RMSEs versus voltage drift, $\mathbf{b}$ Normalised RMSEs based on RMSEs under no voltage drift

RMSE for voltage drift, as shown in Figure 13(b). However, $\mathrm{R}(\mathrm{RQ})$ is still the most accurate FOM in most cases.

The SOC estimation errors of the three FOMs are plotted to visualise the effect of voltage drift on SOC estimation, as shown in Figure 14. One can notice that the results qualitatively coincide with those shown in

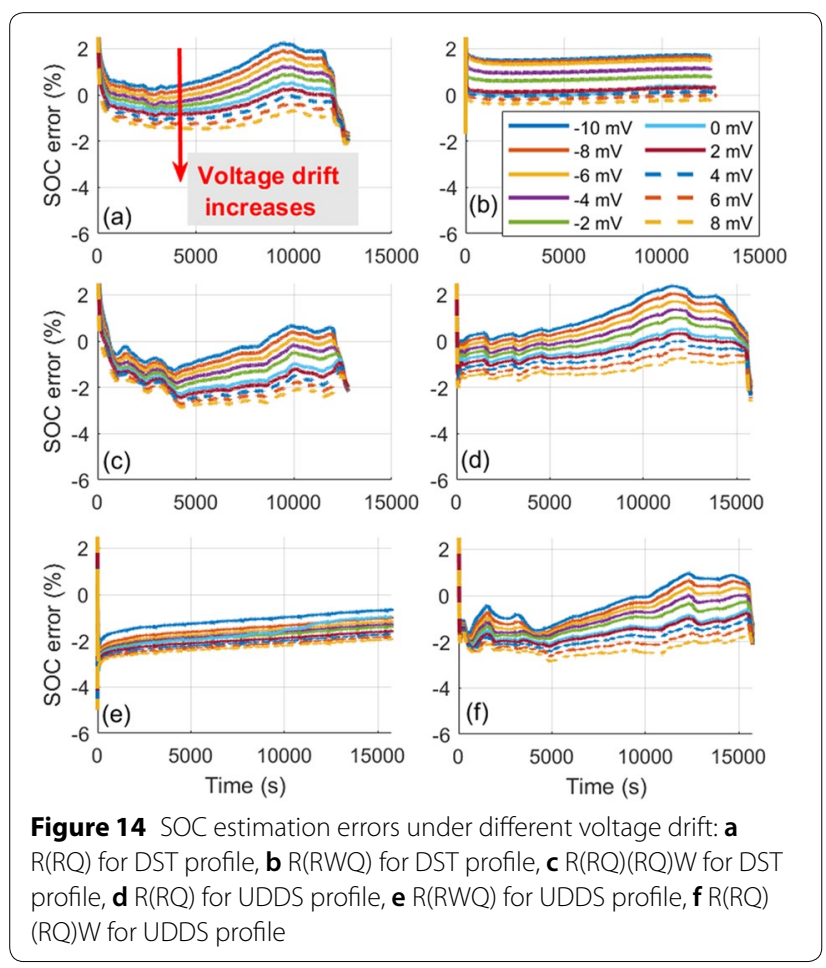

Figure 9, i.e., increasing voltage drift can make SOC estimation error move downward. Thus, both truncation error and voltage drift influence SOC estimation indirectly by affecting voltage simulation accuracy of FOMs. Consequently, we might devise a new SOC estimation framework to adjust memory length to compensate for the effect of voltage drift, which may be an interesting topic for FOMs.

\subsubsection{Influence of Current Sensor Drift}

Different from voltage drift, current drift influences both modelling accuracy and Ampere hour counting algorithm. Zheng et al. [4] suggested that current drift can reach $200 \mathrm{~mA}$ in normal circumstances. To examine the influence of current drift on SOC estimation, we set the current drift to $[-200,-150, \ldots, 200] \mathrm{mA}$ and track the changes of SOC estimation error.

As shown in Figure 15, the current drift has a smaller influence on the RMSEs of all the FOMs than voltage drift but their trends are similar. Moreover, the RMSEs of both $R(R Q)$ and $R(R Q)(R Q) W$ show a linear relationship with current drift, while $R(R W Q)$, instead of $R(R Q)$ in voltage drift, becomes the most sensitive model to current drift. Overall, $R(R Q)$ and $R(R Q)(R Q)$ show the highest and lowest accuracy under various current drifts, respectively.

The SOC estimation errors for all the FOMs are shown in Figure 16. It can be seen that SOC errors accumulate due to the Ampere hour counting method. At the end of the two profiles, the SOC errors based on all the

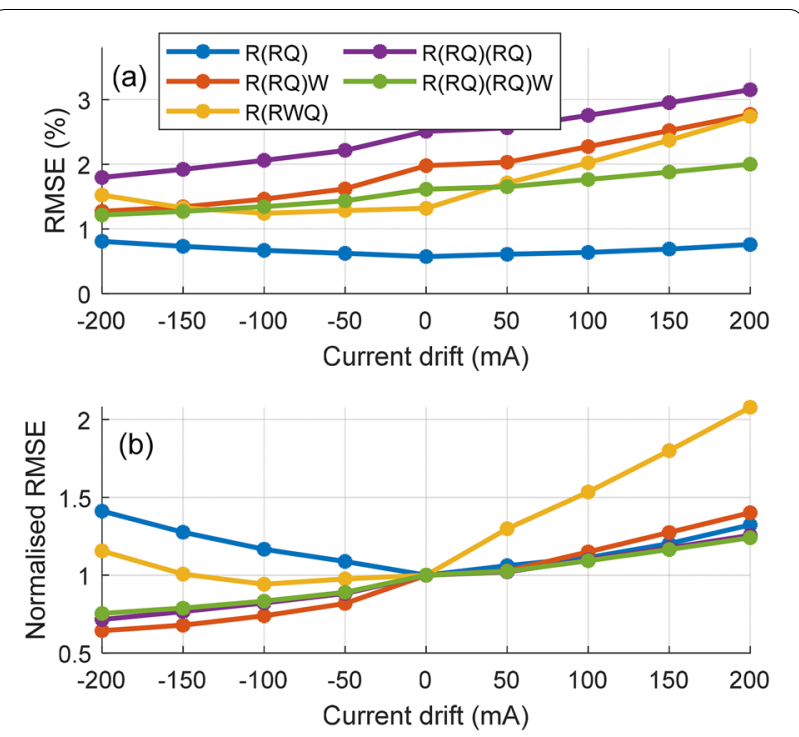

Figure 15 Influence of current drift on SOC estimation error: a RMSE versus current drift, $\mathbf{b}$ Normalised RMSE based on RMSE under no current drift 

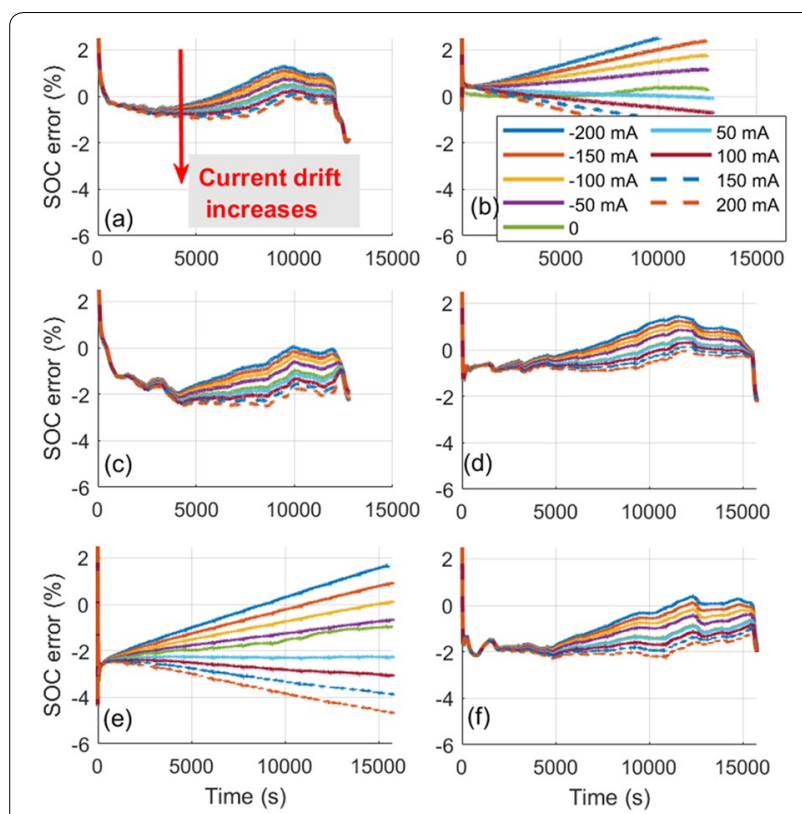

Figure $\mathbf{1 6} \mathrm{SOC}$ estimation errors under different current drifts: a $R(R Q)$ for DST profile, b R(RWQ) for DST profile, $\mathbf{c} R(R Q)(R Q) W$ for $D S T$ profile, $\mathbf{d} R(R Q)$ for UDDS profile, e R(RWQ) for UDDS profile, $\mathbf{f} R(R Q)$ (RQ)W for UDDS profile

FOMs under different current drifts converge, except for $\mathrm{R}(\mathrm{RWQ})$. Therefore, $\mathrm{R}(\mathrm{RWQ})$ is the most vulnerable FOM to current drift although it can keep satisfactory results at the occurrence of voltage drift.

\section{Conclusions}

In this article, five fractional order models (FOMs) for lithium ion batteries are compared in terms of state of charge (SOC) estimation. Offline parameter identification of the five FOMs is conducted first, and the comparisons of the simulated and experimental results show that increasing the complexity of FOMs cannot always improve modelling accuracy. $\mathrm{R}(\mathrm{RQ}) \mathrm{W}$ shows superior identification accuracy than the other four FOMs. Based on the FOMs with the identified parameters, SOC estimation accuracy, complexity and robustness are evaluated under different profiles, memory lengths, temperatures, cells and sensor drifts. The assessment results illustrate that the simplest FOM, R(RQ), has the highest accuracy under normal conditions. The evaluation of the influence of memory length shows that $R(R Q)$ is not sensitive to truncation error, compared with the FOMs with the complex structure. Nevertheless, it has the poorest robustness against the variation of ambient temperature. Validation results on seven cells show the FOMs have good generalisation ability and the $R(R Q)$ shows the smallest error and standard deviation. In the case of current and voltage drifts, all the FOMs can provide satisfactory results over a large range of drift. In particular, $R(R Q)$ has a large relative error when voltage drift happens, but it can still provide the highest accuracy. On the other hand, R(RWQ) is more susceptible to current drift rather than voltage drift, as it cannot weaken the accumulated SOC error.

The work in this article provides a reference for FOM based SOC estimation as FOMs have been illustrated to be feasible for various types of batteries. In the future, we will focus on the application of fractional calculus on battery pack modelling.

\section{Acknowledgements}

The systematic battery tests were performed at the Advanced Energy Storage and Application (AESA) Group, Beijing Institute of Technology. Jinpeng Tian would like to acknowledge the financial support from the China Scholarship Council (CSC).

\section{Authors' Contributions}

JT and RX conceived this study. JT and JW carried out experiments. JT and RX developed the algorithms and wrote the manuscript. RX and WS supervised the study. All authors read and approved the final manuscript.

\section{Authors' Information}

Jinpeng Tian received his B.S. degree in vehicle engineering from Beijing Institute of Technology, China, in 2016, where he is working toward the Ph.D. degree. He is currently studying as a joint Ph.D. candidate at Swinburne University of Technology, Australia. His research interests include modelling and state estimation of lithium-ion batteries. E-mail: tianjinpeng@bit.edu.cn.

Rui Xiong received his M.Sc. degree in vehicle engineering and the Ph.D. degree in mechanical engineering from Beijing Institute of Technology, China, in 2010 and 2014, respectively. He is currently a Professor at Department of Vehicle Engineering, School of Mechanical Engineering, Beijing Institute of Technology, China, where he was appointed to an Associate Professor from 2014. Since 2019, he has been a Visiting Professor at the Massachusetts Institute of Technology, Cambridge, Massachusetts, USA. Since 2017, he has been an Adjunct Professor at the Faculty of Science, Engineering and Technology, Swinburne University of Technology, Melbourne, Vic., Australia. His research interests include electric/ hybrid vehicles, intelligent/connected vehicles, and battery energy storage. E-mail: rxiong@bit.edu.cn.

Weixiang Shen received his Ph.D. degree from University of Hong Kong, Hong Kong, China, in 2002. From 2002 to 2003, he was a Lecturer at Ngee Ann Polytechnic, Singapore. From 2003 to 2008, he was a Lecturer and then a Senior Lecturer at School of Engineering, Monash University Malaysia. He then worked as a Research Fellow for one year at School of Electrical and Electronics Engineering, Nanyang Technological University, Singapore. Currently, he is an Associate Professor in Electrical Engineering at Faculty of Science, Engineering and Technology, Swinburne University of Technology, Australia. His research interests include battery charging, battery capacity estimation, battery fault diagnosis and battery management systems for electric vehicles and integration of renewable energy sources into power grids. E-mail: wshen@swin.edu.au.

Ju Wang received his Ms.E. degree in automotive engineering from Beijing Institute of Technology, China, in 2017. He is currently working toward the Ph.D. degree in mechanical engineering with the National Engineering Laboratory for Electric Vehicles, Beijing Institute of Technology and Collaborative Innovation Center of Electric Vehicles in Beijing, China. His research mainly focuses on multistate estimation of lithium ion batteries and battery management system design. E-mail: wang_ju@bit.edu.cn.

\section{Funding}

Supported by Beijing Municipal Natural Science Foundation of China (Grant No. 3182035) and National Natural Science Foundation of China (Grant No. 51877009). 


\section{Competing Interests}

The authors declare no competing financial interests.

\section{Author Details}

${ }^{1}$ Department of Vehicle Engineering, School of Mechanical Engineering, Beijing Institute of Technology, Beijing 100081, China. ${ }^{2}$ Faculty of Science, Engineering and Technology, Swinburne University of Technology, Hawthorn, Victoria 3122, Australia.

Received: 17 April 2020 Revised: 23 June 2020 Accepted: 28 June 2020 Published online: 13 July 2020

\section{References}

[1] M A Hannan, M S H Lipu, A Hussain, et al. A review of lithium-ion battery state of charge estimation and management system in electric vehicle applications: Challenges and recommendations. Renew. Sustain. Energy Rev., 2017, 78: 834-854.

[2] W Wang, Y Li, C Lin, et al. Mass-spring-damping theory based equivalent mechanical model for cylindrical lithium-ion batteries under mechanical abuse. Chinese J. Mech. Eng, 2020, 33:23.

[3] Y Sun, Z Ma, G Tang, et al. Estimation method of state-of-charge for lithium-ion battery used in hybrid electric vehicles based on variable structure extended Kalman filter. Chinese J. Mech. Eng, 2016, 29(4): 717-726.

[4] $Y$ Zheng, M Ouyang, $X$ Han, et al. Investigating the error sources of the online state of charge estimation methods for lithium-ion batteries in electric vehicles. J. Power Sources, 2018, 377: 161-188.

[5] M Doyle, T F Fuller, J S Newman. Modeling of galvanostatic charge and discharge of the lithium/polymer/insertion cell. J. Electrochem. Soc, 1993, 140(6): 1526-1533.

[6] TF Fuller, M Doyle, J Newman. Simulation and optimization of the dual lithium ion insertion cell. J. Electrochem. Soc, 1994, 141(1): 1-10.

[7] X Kong, G L Plett, M S Trimboli, et al. Pseudo-two-dimensional model and impedance diagnosis of micro internal short circuit in lithium-ion cells. J. Energy Storage, 2020, 27: 101085.

[8] R Xiong, S Ma, H Li, et al. Toward a safer battery management system: A critical review on diagnosis and prognosis of battery short circuit. iscience, 2020, 23(4): 101010.

[9] M R Palacín. Understanding ageing in Li-ion batteries: A chemical issue. Chem. Soc. Rev., 2018, 47(13): 4924-4933.

[10] J Tian, R Xiong, W Shen. A review on state of health estimation for lithium ion batteries in photovoltaic systems. eTransportation, 2019, 2: 100028.

[11] R Xiong, Y Zhang, J Wang, et al. Lithium-ion battery health prognosis based on a real battery management system used in electric vehicles. IEEE Trans. Veh. Technol, 2019, 68(5): 4110-4121.

[12] P Amiribavandpour, W Shen, D Mu, et al. An improved theoretical electrochemical-thermal modelling of lithium-ion battery packs in electric vehicles. J. Power Sources, 2015, 284: 328-338.

[13] C Lin, A Tang. Simplification and efficient simulation of electrochemical model for li-ion battery in EVs. Energy Procedia, 2016, 104: 68-73.

[14] R Xiong, J Tian, H Mu, et al. A systematic model-based degradation behavior recognition and health monitoring method for lithium-ion batteries. Appl. Energy, 2017, 207: 372-383.

[15] P Shrivastava, T Kok, M Yamani, et al. Overview of model-based online state-of-charge estimation using Kalman filter family for lithium-ion batteries. Renew. Sustain. Energy Rev., 2019, 113: 109233.

[16] Y Zheng, W Gao, X Han, et al. An accurate parameters extraction method for a novel on-board battery model considering electrochemical properties. J. Energy Storage, 2019, 24: 100745.

[17] R Xiong, Q Yu, W Shen, et al. A sensor fault diagnosis method for a lithium-ion battery pack in electric vehicles. IEEE Trans. Power Electron, 2019, 34(10): 9709-9718.

[18] R Xiong, R Yang, Z Chen, et al. Online fault diagnosis of external short circuit for lithium-ion battery pack. IEEE Trans. Ind. Electron, 2020, 67(2): 1081-1091.

[19] H He, R Xiong, J Fan. Evaluation of lithium-ion battery equivalent circuit models for state of charge estimation by an experimental approach. Energies, 2011, 4(4): 582-598.
[20] X Lai, Y Zheng, T Sun. A comparative study of different equivalent circuit models for estimating state-of-charge of lithium-ion batteries. Electrochim. Acta, 2018, 259: 566-577.

[21] J Huang, Z Li, B Y Liaw, et al. Graphical analysis of electrochemical impedance spectroscopy data in Bode and Nyquist representations. J. Power Sources, 2016, 309: 82-98.

[22] H Qu, J Kafle, J Harris, et al. Application of ac impedance as diagnostic tool - Low temperature electrolyte for a Li-ion battery. Electrochim. Acta, 2019, 322: 134755.

[23] T Osaka, D Mukoyama, H Nara. Review-development of diagnostic process for commercially available batteries, especially lithium ion battery, by electrochemical impedance spectroscopy. J. Electrochem. Soc, 2015, 162(14): A2529-A2537.

[24] S Guo, R Xiong, W Shen, et al. Aging investigation of an echelon internal heating method on a three-electrode lithium ion cell at low temperatures. J. Energy Storage, 2019, 25(5): 100878.

[25] H Ge, J Huang, J Zhang, et al. Temperature-adaptive alternating current preheating of lithium-ion batteries with lithium deposition prevention. J. Electrochem. Soc, 2016, 163(2): A290-A299.

[26] B Hirschorn, M E Orazem, B Tribollet, et al. Determination of effective capacitance and film thickness from constant-phase-element parameters. Electrochim. Acta, 2010, 55(21): 6218-6227.

[27] J Huang, H Ge, Z Li, J Zhang. Dynamic electrochemical impedance spectroscopy of a three-electrode lithium-ion battery during pulse charge and discharge. Electrochim. Acta, 2015, 176: 311-320.

[28] X Lu, H Li, N Chen. An indicator for the electrode aging of lithium-ion batteries using a fractional variable order model. Electrochim. Acta, 2019, 299: 378-387.

[29] D Andre, M Meiler, K Steiner, et al. Characterization of high-power lithiumion batteries by electrochemical impedance spectroscopy. II: Modelling. J. Power Sources, 2011, 196(12): 5349-5356.

[30] M Hu, Y Li, S Li, et al. Lithium-ion battery modeling and parameter identification based on fractional theory. Energy, 2018, 165: 153-163.

[31] S Li, M Hu, Y Li, et al. Fractional-order modeling and SOC estimation of lithium-ion battery considering capacity loss. Int. J. Energy Res., 2019, 43(1): 417-429.

[32] J Xu, C C Mi, B Cao, et al. A new method to estimate the state of charge of lithium-ion batteries based on the battery impedance model. J. Power Sources, 2013, 233: 277-284.

[33] C Liu, W Liu, L Wang, et al. A new method of modeling and state of charge estimation of the battery. J. Power Sources, 2016, 320: 1-12.

[34] C Zou, X Hu, S Dey, et al. Nonlinear fractional-order estimator with guaranteed robustness and stability for lithium-ion batteries. IEEE Trans. Ind. Electron, 2017, 65(7): 5951-5961.

[35] R Xiong, J Tian. A comparative study on fractional order models for voltage simulation of lithium ion batteries. IEEE 89th Veh. Technol. Conf., IEEE، 2019: 1-5.

[36] R Yang, $\mathrm{R}$ Xiong, $\mathrm{H}$ He, et al. A fractional-order model-based battery external short circuit fault diagnosis approach for all-climate electric vehicles application. J. Clean. Prod, 2018, 187: 950-959.

[37] Q Yang, J Xu, B Cao, et al. A simplified fractional order impedance model and parameter identification method for lithium-ion batteries. PLoS One, 2017, 12(2): 1-13.

[38] X Hu, H Yuan, C Zou, et al. Co-estimation of state of charge and state of health for lithium-ion batteries based on fractional-order calculus. IEEE Trans. Veh. Technol, 2018, 67(11): 10319-10329.

[39] Y Chen, D Huang, Q Zhu, et al. A new state of charge estimation algorithm for lithium-ion batteries based on the fractional unscented Kalman filter. Energies, 2017, 10(9): 1313.

[40] B Wang, Z Liu, S E Li, et al. State-of-charge estimation for lithium-ion batteries based on a nonlinear fractional model. IEEE Trans. Control Syst. Technol., 2017, 25(1): 3-11.

[41] J Tian, R Xiong, Q Yu. Fractional-order model-based incremental capacity analysis for degradation state recognition of lithium-ion batteries. IEEE Trans. Ind. Electron, 2019, 66: 1576-1584.

[42] Y Zou, S E Li, B Shao, et al. State-space model with non-integer order derivatives for lithium-ion battery. Appl. Energy, 2016, 161: 330-336.

[43] L De Sutter, Y Firouz, J De Hoog, et al. Battery aging assessment and parametric study of lithium-ion batteries by means of a fractional differential model. Electrochim. Acta, 2019, 305: 24-36. 
[44] Y Jiang, B Xia, X Zhao, et al. Data-based fractional differential models for non-linear dynamic modeling of a lithium-ion battery. Energy, 2017, 135: 171-181.

[45] H Mu, R Xiong, $\mathrm{H}$ Zheng, et al. A novel fractional order model based stateof-charge estimation method for lithium-ion battery. Appl. Energy, 2017, 207: 384-393

[46] J Peng, J Luo, H He, et al. An improved state of charge estimation method based on cubature Kalman filter for lithium-ion batteries. Appl. Energy, 2019, 253: 113520.

[47] I Petráš. Fractional-order nonlinear systems: modeling, analysis and simulation. Springer Science \& Business Media, 2011.

[48] Q Yu, R Xiong, L Y Wang, et al. A comparative study on open circuit voltage models for lithium-ion batteries. Chinese J. Mech. Eng., 2018, 31: 65.

[49] R Xiong, J Tian, W Shen, et al. A novel fractional order model for state of charge estimation in lithium ion batteries. IEEE Trans. Veh. Technol., 2019, 68(5): 4130-4139.
[50] R Caballero-Águila, A Hermoso-Carazo, J Linares-Pérez. Extended and unscented filtering algorithms in nonlinear fractional order systems with uncertain observations. Appl. Math. Sci., 2012, 6: 1471-1486.

[51] C Chen, R Xiong, R Yang, et al. State-of-charge estimation of Lithium-ion battery using an improved neural network model and extended Kalman filter. J. Clean. Prod, 2019, 234: 1153-1164.

[52] X Liu, Z Chen, C Zhang, et al. A novel temperature-compensated model for power Li-ion batteries with dual-particle-filter state of charge estimation. Appl. Energy, 2014, 123: 263-272.

[53] Y Zheng, M Ouyang, L Lu, et al. Cell state-of-charge inconsistency estimation for LiFePO4 battery pack in hybrid electric vehicles using meandifference model. Appl. Energy, 2013, 111: 571-580.

[54] L Lu, X Han, J Li, et al. A review on the key issues for lithium-ion battery management in electric vehicles. J. Power Sources, 2013, 226: 272-288. 